Pacific Journal of Mathematics

AUTOMORPHISM GROUPS OF UNIPOTENT GROUPS O 


\title{
AUTOMORPHISM GROUPS OF UNIPOTENT GROUPS OF CHEVALLEY TYPE
}

\author{
A. Fauntleroy
}

Let $G$ be a quasi-simple algebraic group defined and split over the field $k$. Let $V$ be a maximal $k$-split unipotent subgroup of $G$ and $\operatorname{Aut}(V)$ the group of $k$-automorphism of $V$. The structure of Aut $(V)$ is determined and the obstructions to making $\operatorname{Aut}(V)$ algebraic when char $k>3$ are made explicit. If $G$ is not of type $A_{2}$, then $\operatorname{Aut}(V)$ is solvable.

Introduction. In [5] Hochschild and Mostow showed that the automorphism group of a unipotent algebraic group defined over a field $k$ of characteristic zero carries the structure of an algebraic $k$-group. For example if $V$ is a vector group over $\mathbf{C}$, then $\operatorname{Aut}_{\mathbf{C}}(V)=\mathrm{GL}(n, \mathbf{C})$. For more complicated unipotent groups - even over $\mathbf{C}$ - little seems to be known about the actual structure of the automorphism group. On the other hand, it was shown by Sullivan in [8] and again by this author in [3] that the Hochschild-Mostow result never holds in positive characteristics when the dimension of the given unipotent group is greater than one.

In [4] Gibbs determined generators for the (abstract) automorphism group of $V(k)$ - the $k$-rational points of a maximal $k$-split unipotent subgroup $V$ of any $k$-split simple algebraic group. The characteristic of the field $k$ was assumed distinct from 2 or 3 , but no other assumptions on the field $k$ were made. We refer to such groups $V$ as unipotent groups of Chevalley type. The purpose of this paper is two-fold:

1. To determine the automorphism groups in characteristic zero of unipotent groups of Chevalley type; and

2. To exhibit the obstructions to making these groups algebraic in positive characteristics.

Let $A u t_{v}(k)$ denote the group of $k$-automorphisms of the unipotent $k$-group of Chevalley type $V$. We show (2.9) that there is an exact sequence

$$
1 \rightarrow N(k) \rightarrow A u t_{V}(k) \rightarrow H(k) \rightarrow 1
$$

such that $H$.

(i) $H(k)$ is the group of $k$-rational points of an algebraic $k$-group

(ii) $N(k)=0$ if char $k=0$, and $N(k)=\amalg_{n=1}^{\infty} G_{a}(k)$ if char $k>3$.

(iii) The above sequence splits and $\operatorname{Aut}_{v}(k)$ is the semi-direct product of $N(k)$ and $H(k)$. 
Moreover, if the quasi-simple group containing $V$ is not isogenous to $\mathrm{PGL}_{2}$, then $A u t_{V}(k)$ is solvable.

Our treatment of this problem is slightly more general than required to prove the desired results over fields. In fact if $A=\mathbf{Z}[1 / 2,1 / 3]$, then all schemes (no separation property is implied here) are assumed to be $A$-schemes and the problem we discuss is that of representing the functor $S \rightarrow A u t_{V}(S):=\operatorname{Aut}_{s-g r}\left(V \times_{A} S\right)$ for $S$ a reduced $A$-scheme. The results for fields then follow by base change.

After setting up some notation and discussing preliminaries in $§ 0$, we proceed in $\$ 1$ to give a functorial description of the generators described by Gibbs. Section 2 is devoted to the computation of $A u t_{V}$ and $\S 3$ to the special case of groups of type $\boldsymbol{A}_{2}$.

The author's debt to Gibbs' work will become clear soon. It is also a pleasure to thank J. Tits for several useful comments on an earlier version of this paper.

\section{Preliminaries.}

0.1 . Let $\mathscr{L}$ be a simple complex Lie algebra with root system $\Sigma$ and fundamental roots $\Phi \subset \Sigma$. There is a unique (up to isomorphism) smooth algebraic group scheme ch $(\Phi, \Sigma)$, defined over the integers, corresponding to $\mathscr{L}$ called the Chevalley group of type $\Sigma$ [2: Vol III, Exp. $\mathrm{XXV}$. If the set of positive roots relative to $\Phi$ is denoted $\Sigma^{+}$, then $\Sigma^{+}$ determines a unique Borel subgroup $B=B\left(\Sigma^{+}\right)$of $\operatorname{ch}(\Phi, \Sigma)$. Let $V=$ $V(\Phi, \Sigma)$ be the unipotent radical of $B$. We call $V$ a unipotent group scheme of Chevalley type.

0.2. A unipotent group scheme of Chevalley type, say $V$, is completely determined by the group valued functor it represents. We recall the definition here. Let $S$ be a scheme. Then $V(S)$ as an abstract group is generated by symbols $x_{r}(t), r \in \Sigma^{+}, t \in \Gamma\left(S, \theta_{S}\right):=$ $G_{a}(S)$ subject to the relations

$$
\text { R.1: } x_{r}(t) x_{r}(u)=x_{r}(t+u)
$$

$$
\text { R.2: }\left[x_{s}(u), x_{r}(t)\right]= \begin{cases}1 & r+s \notin \Sigma^{+} \\ \prod_{i, j} x_{i r+j s}\left(C_{i j, r s}(-t)^{i} u^{\prime}\right) & r+s \in \Sigma^{+}\end{cases}
$$

where the product in R.2 is taken over all pairs of positive integers $(i, j)$ such that $i r+j s \in \Sigma^{+}$. We use the conventions: $[a, b]=a^{-1} b^{-1} a b$ and (int $a) b=a^{-1} b a$.

0.3 . With respect to a given ordering of $\Sigma^{+}$, every element of $V(S)$ can be written uniquely in the form 


$$
x_{r_{1}}\left(t_{1}\right) \cdot x_{r_{2}}\left(t_{2}\right) \cdots x_{r_{N}}\left(t_{N}\right)
$$

where $N=\left|\Sigma^{+}\right|$and $r_{1}<r_{2}<\cdots<r_{N}$. Moreover, the constants $C_{i, r s}$ are integers and $\left|C_{i j, r s}\right|<4$. Recall that $N_{r s}=C_{11, r s}$, and also that $C_{12, r s}=$ $\frac{1}{2} N_{s r} N_{s, r+s}, C_{21, r s}=\frac{1}{2} N_{r s} N_{r, r+s}, N_{r s}=-N_{s r}$ and $N_{r s}= \pm 1$ all $r, s$ in $\Sigma$.

0.4. The subgroups $X_{r}, r \in \Sigma^{+}$given by $X_{r}(S)=\left\{x_{r}(t): t \in G_{a}(S)\right\}$ are called root subgroups and each $X_{r} \simeq G_{a}$. The uniqueness assertion of 0.3 says that the product morphism $X_{r_{1}} \times \cdots \times X_{r_{N}} \rightarrow V$ is an isomorphism.

0.5. Put $V_{m}=\prod_{h(r) \geqq m} X_{r}$ for $1<m \leqq h=h\left(r_{N}\right)$ where $h(r)$ is the height function (c.f. $[4 ; \S 0]$ ).

Proposition. [4: 5.4] The series

$$
V=V_{1}>V_{2}>\cdots>V_{h}>1
$$

is both the upper and lower central series for $V$.

Moreover, for each $i, V_{\downarrow} / V_{t+1}$ has a canonical structure of a vector group (i.e. is isomorphic to $G_{a}^{n}$ for some integer $n$ ).

0.6. In the following we exclude the trivial case $\Sigma=A_{1}$.

Proposition. Let $\left(\Sigma, \Phi, \Sigma^{+}\right)$be a system of roots, fundamental roots and positive roots in a complex simple Lie algebra. Then

(i) There exist a unique root $r_{N} \in \Sigma^{+}$of maximal height $h$.

(ii) If $r \in \Sigma^{+}$and $h(r) \geqq 2$ then there exist $r_{\imath} \in \Phi$ such that $r-r_{1} \in \Sigma^{+}$.

(iii) If $r \in \Sigma^{+}$and $r \neq r_{N}, r \notin \Phi$ then there exist $r_{i} \in \Phi$ such that $r+r_{i} \in \Sigma^{+}$.

(iv) If the combination ir $+j$ for $i>0, j>0, r, s \in \Sigma^{+}$lies in $\Sigma^{+}$then $r+s \in \Sigma^{+}$.

(v) There exist $r_{i} \in \Phi$ such that $r_{N}-r_{\imath} \in \Sigma^{+}$.

We make some remarks concerning (v). It is easy to see by examining the root systems that, if $\Sigma$ is not of type $A_{l}$, then there is a unique fundamental root $r_{l} \in \Phi$ with $r_{N}-r_{l} \in \Sigma^{+}$. If $\Sigma$ is of type $C_{l}$ and $r_{N}-r_{i} \in \Sigma^{+}$, then $r_{N}-2 r_{i}$ is also in $\Sigma^{+}$. In particular, if $\Sigma$ is not of type $A_{l}$, then there is a unique root of height $h-1$. For if $h(r)=h-1$, $r \in \Sigma^{+}$, then $h\left(r+r_{l}\right)=h$ some $\cdot r_{j} \in \Sigma^{+}$by (iii) so that $r+r_{j}=r_{N}$ by (i). Thus $r_{N}-r_{j}=r \in \Sigma^{+}$, so $r_{j}=r_{i}$.

If $\Sigma$ is of type $C_{l}$ and $h(s)=h-2$, then $X_{s}$ and $X_{r \sim 2 r_{i}}$ commute. For $h\left(s+r_{N}-2 r_{\imath}\right)=2 h-4$. But in this case $h=h\left(r_{N}\right) \geqq 5$ 
so that $2 h-4>h$ and, hence, $s+r_{N}-2 r_{i} \notin \Sigma^{+}$. So by (iv) and R.2, $X_{s}$ and $X_{r N-2 r_{i}}$ commute. It follows that we can assume that, in case $C_{l}$, the roots are ordered so that $r_{N}-2 r_{i}, r_{N}-r_{i}$ and $r_{N}$ are the last three roots. We keep this assumption throughout.

If $\Sigma$ is of type $A_{l}$ with $l>2$, then there are just two roots $r_{1}, r_{l} \in \Phi$ such that $r_{N}-r_{i} \in \Sigma^{+}, \quad i=1, l$. In this case, since $h\left(r_{N}\right)>2$, $r_{N}-r_{1}+r_{N}-r_{l}$ is not a root so that $X_{r_{N}-r_{1}}$ and $X_{r_{N}-r}$ commute. This fails of course if $\Sigma=A_{2}$ for then $\Sigma^{+}=\{r, s, r+s\}$.

In what follows we set $A:=Z[1 / 2,1 / 3]$. All schemes are $A$ schemes. We assume no separation property for $A$-schemes.

1. The Gibbs subfunctors. In [4] Gibbs listed six types of automorphisms of the abstract group $V(k)$ for any field and showed that every automorphism was a product of these six types. We shall give here five of those six types - described functorially - which will in fact generate $A_{u t}(S)$ for any reduced $A$-scheme $S$.

1.1. Diagonal automorphisms. Let $M$ be the free abelian group generated by the set of roots $\Phi \subset \Sigma$ determining $V=V(\Phi, \Sigma)$. Let $D$ be the $A$-torus representing $D_{A}(M)$. Recall $D_{A}(M)$ is the functor defined by

$$
D_{A}(M)(S)=\operatorname{Hom}_{A-\text { alg }}\left(A[M], \Gamma\left(S, O_{S}\right)\right)
$$

where $A[M]$ is the group algebra of $M$ over $A$.

We define a homomorphism of group valued functors

$$
w_{D}: h_{D}=D(M) \rightarrow A_{u t}
$$

as follows: For any $A$-scheme $S$ and $\lambda \in h_{D}(S)$ let $w_{D}(\lambda)$ be the map which sends $x_{r}(t)$ to $x_{r}(\lambda(r) t)$ all $r \in \Sigma^{+}$. As in [4: §4], it is easily verified that this determines an automorphism of $V(S)$.

Since $\lambda(r) \in \Gamma\left(S, O_{S}\right)^{*}$, it is also easy to see that $w_{D}(\lambda)$ is in fact an automorphism of $V \times_{A} S$ over $S$. Indeed, if $B$ is the standard Borel subgroup of $\operatorname{Ch}(\Phi, \Sigma)$ determined by $\Sigma^{+}$and $T$ is its maximal torus, then (cf. [2: Vol. III, Exp. XXII, 1.13]) $D \cong T$ and $w$ is just the map induced by $T$ acting on $V \cong B_{u}$ via conjugation. In particular, $w_{D}$ is a homomorphism of group valued functors which (since $\operatorname{Ch}(\Phi, \Sigma)$ is of adjoint type) is in fact a monomorphism.

1.2. Inner automorphisms. Let $I=V / Z(V)$ where $Z(V)$ is the center of $V$. Then we have the natural functorial monomorphism of group valued functors $I \rightarrow A u t_{V}$. 
1.3. Central automorphisms. Let $\Lambda$ be the functor from $\mathrm{Sch} / A$ to abelian groups given by $\Lambda(S)=\operatorname{Hom}_{s-\mathrm{gr}}\left(G_{a} \times S, G_{a} \times S\right)$ and let $l=$ $|\Phi|$. Put $\mathbf{C}=\Lambda \times \cdots \times \Lambda$ ( $l$ copies). We define a monomorphism $w_{\mathrm{C}}: \mathbf{C} \rightarrow A u t_{V}$ of group valued functors as follows:

For $S \in \mathrm{Sch} / A$ and $c=\left(c_{1}, \cdots, c_{l}\right) \in \mathbf{C}(S)$ we define $w_{C}(c)$ by

$$
\begin{aligned}
w_{\mathrm{C}}(c)[ & \left.x_{r_{1}}\left(t_{1}\right) \cdots x_{r_{N}}\left(t_{N}\right)\right] \\
& =x_{r_{1}}\left(t_{1}\right) \cdots x_{r_{N-1}}\left(t_{N-1}\right) x_{r N}\left(t_{N}+\sum_{i=1}^{l} c_{i}\left(t_{t}\right)\right)
\end{aligned}
$$

for all $t_{t} \in \Gamma\left(S, O_{S}\right), 1 \leqq i \leqq N$ and $c_{\imath} \in \operatorname{End}_{s-\mathrm{gr}}\left(G_{a} \times{ }_{A} S\right)$ where $c_{\imath}\left(t_{t}\right)$ is given by the canonical action of $c_{i}$ on $\Gamma\left(S, O_{S}\right)=G_{a}(S)$. Since the subgroup $X_{r_{\mathrm{N}}}$ is central, it follows immediately that $w_{\mathrm{C}}(c)$ is an automorphism of $V(S)$ and it is equally clear that $w_{\mathrm{C}}(c)$ yields an element of $\operatorname{Aut}_{\mathrm{V}}(\mathrm{S})$.

Finally, recall that the abelian group structure on $\Lambda(S)$ is given by $\left(c_{1}+c_{2}\right)(t)=c_{1}(t)+c_{2}(t)$ all $t \in G_{a}(S)$; so $w_{C}$ is in fact a monomorphism of group valued functors.

1.4. Graph automorphisms. Let $\Pi$ be a finite group of automorphisms of $M$ which stabilize $\Phi$ and hence $\Sigma^{+}$. Then an element $g \in \Pi$ determines a graph automorphism $w_{\Pi}(g)$ of $V \times_{A} S$ over $S$ for an $A$-scheme $S$ via the assignments $x_{r}(t) \rightarrow x_{g(r)}(t)$ all $r \in \Sigma^{+}$and $t \in$ $\Gamma\left(S, O_{s}\right)$. Such automorphisms arise as certain graph automorphisms of $\operatorname{Ch}(\Phi, \Sigma)$ determined by Steinberg [1: 12.2] which stabilize $\Phi$.

If $\Sigma$ is of type $A_{l}(l>1), D_{l}(l>4)$ or $E_{6}$ then $\Pi=Z_{2}$. If $\Sigma$ is of type $D_{4}$, then $\Pi=S_{3}$. These are the only graph automorphisms which occur. We identify $\Pi$ with the constant functor and see immediately that $w_{\Pi}$ is a monomorphism of group valued functors.

1.5. Extremal automorphisms. Let $r_{N}$ be the unique root in $\Sigma^{+}$of maximal height (cf. 0.6). Then there is a root $r_{i} \in \Phi$ such that $r_{N}-r_{i} \in$ $\Sigma^{+}$. Suppose temporarily that $\Sigma$ is not of type $A_{l}$ or $C_{l}$. Put $E=G_{a}$ and define a map $w_{E}: E \rightarrow A u t_{V}$ as follows: For any $A$-scheme $S$ and $u \in E(S)=\Gamma\left(S, O_{S}\right)$ let $w_{E}(u)$ be the map which acts trivially on $x_{r}(t)$, $r \neq r_{i}$ and which sends $x_{r_{i}}(t)$ to

$$
x_{r_{i}}(t) x_{r N-r_{i}}(u t) x_{r N}\left(\frac{1}{2} N_{r N-r_{r} i} u t^{2}\right)
$$

for all $t \in \Gamma\left(S, O_{S}\right)$. As in [4: §4] one verifies that $w_{E}(u)$ determines an automorphism of $V(S)$. It is also seen that this induces a (unique) automorphism of $V \times_{A} S$ over $S$. The following lemma is immediate.

LEMMA 1.6. The map $w_{E}: E \rightarrow A u t_{V}$ is a monomorphism of group valued functors. 
Suppose now that $\Sigma$ is of type $A_{l}(l>1)$. Then there are two fundamental roots, say $r_{1}$ and $r_{l}$, such that $r_{N}-r_{i} \in \Sigma^{+}$. The lemma shows that for each $r_{i}, i=1, l$ we have a monomorphism $w_{i}: G_{a} \rightarrow A u t_{v}$. If $l>2$ then $r_{N}-r_{1} \neq r_{l}$ and $r_{N}-r_{l} \neq r_{1}$. Since $X_{r_{n}-r_{2}}$ and $X_{r_{n}-r_{l}}$ commute if $l>2$, for all $u, v \in G_{a}(S)$ we have $w_{1}(u) w_{l}(v)=$ $w_{l}(v) w_{1}(u)$. We thus obtain a monomorphism $w_{E}: E=$ $G_{a} \times G_{a} \rightarrow A u t_{v}$. When $l=2$ we have two monomorphisms $w_{1}$ and $w_{2}$ (recall in this case $\Sigma=\{r, s, r+s\}$ ) and we denote by $E$ the subfunctor of $A u t_{V}$ which the images of these two homomorphisms generate. We will see in $\S 3$ that this case gives rise to the only exception to the solvability of $A u t_{V}$.

Now suppose $\Sigma$ is of type $C_{l}$. Then if $r_{N}-r_{1} \in \Sigma^{+}$, we also have $r_{N}-2 r_{\imath} \in \Sigma^{+}$. We have just as above $w_{1}: G_{a} \rightarrow A u t_{V}$; but we can also define another map $w_{2}: G_{a} \rightarrow A u t_{V}$ by $w_{2}(u)\left[x_{r}(t)\right]=x_{r}(t), r \neq r_{t}$, and

$$
\begin{gathered}
w_{2}(u)\left[x_{r_{i}}(t)\right]=x_{r_{i}}(t) x_{r_{N}-2 r_{i}}(u t) x_{r_{N}-r_{i}}\left(\frac{1}{2} N_{r_{N}-2 r_{i} r_{i}} u t^{2}\right) \\
\cdot x_{r_{N}}\left(\frac{1}{3} C_{12, r_{N}-2 r_{r_{r}} r_{i}} u t^{3}\right) .
\end{gathered}
$$

As above, the following lemma follows from straight forward computations.

LEMMA 1.7. The morphism $w_{2}$ is a monomorphism of group valued functors and $w_{2}(u) w_{1}(v)=w_{1}(v) w_{2}(u)$ for all $u, v \in G_{a}(S), \quad S \in$ $\mathrm{Sch} / k$. In particular there exist a monomorphism of group valued functors

$$
w_{E}: E=G_{a} \times G_{a} \rightarrow A u t_{V}
$$

such that $w_{E}(u, 0)=w_{1}(u)$ and $w_{E}(0, v)=w_{2}(v)$.

2. The structure of $\boldsymbol{A} \boldsymbol{u} t_{v}$. The purpose of this section is to describe the functor $A u t_{V}$ in terms of the Gibbs subgroups and prove that these generate when $A u t_{V}$ is restricted to the category $(\mathrm{Sch} / A)_{\text {red }}$ of reduced $A$-schemes. Let $\mathbf{A}_{V}$ be the subgroup of $A u t_{V}$ generated by $\pi, D, E, I$ and $C$. We assume throughout this section that $\Sigma \neq A_{2}, B_{2}$. We let $V$ be a unipotent group of Chevalley type over $A$ and $S$ an $A$-scheme.

\section{Proposition 2.1. The subgroup $\mathbf{C}$ is normal in $\mathrm{Aut}$.}

Proof. Recall that if $U$ is any abstract group, $U^{\prime}$ its commutator subgroup and $Z$ its center then there is a well-known homomorphism of monoids $\alpha: \operatorname{Hom}\left(U / U^{\prime}, Z\right) \rightarrow \operatorname{End}(U)$ defined by $\alpha(h)(u)=$ $u h\left(u U^{\prime}\right)$. Since $A u t(U)$ operates on all objects involved and clearly preserves $\alpha$, the intersection $\operatorname{Im} \alpha \cap \operatorname{Aut}(U)$ is a normal subgroup of $\operatorname{Aut}(U)$. 
Applying this to our case set $U=V(S), U^{\prime}=V_{2}(S) Z=V_{h}(S)$ (c.f. 0.5). Then $U / U^{\prime} \simeq G_{a}(S)^{l}$ and the map $\alpha$ is just the map $w_{C}$ defined in 2.3. Since $Z \subset U^{\prime}$ it follows that $\operatorname{Im} \alpha \cap$ Aut $U=C(S)$ is normal in Aut $U$.

Proposition 2.2. Let $\bar{W}$ be the subfunctor of $\mathbf{A}_{V}$ generated by $E, I$ and C. Then $D$ normalizes $\bar{W}$ and $D \bar{W}$ is a semidirect product.

Proof. Since $D$ normalizes $\mathbf{C}$ and $I$ is normal in $A u t_{V}$ it suffices to show $D$ normalizes $E$.

Let $r_{i} \in \Phi$ and $r_{N}-r_{i} \in \Sigma^{+}$. Let $e \in E(S)$ be the extremal automorphism determined by $x_{r_{i}}(t) \rightarrow x_{r_{i}}(t) x_{r_{N}-r_{i}}(u t) x_{r N}\left(K u t^{2}\right), K$ an appropriate constant and suppose $d \in D(S)$ corresponds to the character $\alpha$.

Now $\operatorname{ded}^{-1}\left[x_{r_{i}}(t)\right]=\operatorname{de}\left[x_{r_{i}}\left(\alpha\left(r_{i}\right)^{-1} t\right)\right]$. Put $s=\alpha\left(r_{i}\right)^{-1} t$. Then

$$
\begin{aligned}
d e\left[x_{r_{i}}(s)\right] & =d\left[x_{r_{i}}(s) x_{r_{N} r_{t}}(u s) x_{r_{N}}\left(K u s^{2}\right)\right] \\
& =x_{r_{i}}\left(\alpha\left(r_{t}\right) s\right) x_{r_{N}-r_{i}}\left(\alpha\left(r_{N}-r_{t}\right) u s\right) x_{r_{N}}\left(K \alpha\left(r_{N}\right) u s^{2}\right) .
\end{aligned}
$$

Since $\alpha$ is a character $\alpha\left(r_{N}-r_{i}\right)=\alpha\left(r_{N}\right) \alpha\left(r_{i}\right)^{-1}$. Thus

$$
\operatorname{ded}^{-1}\left[x_{r_{i}}(t)\right]=x_{r_{i}}(t) x_{r_{N} r_{i}}\left(u^{\prime} t\right) x_{r N}\left(K u^{\prime} t^{2}\right)
$$

where $u^{\prime}=\left(\alpha\left(r_{N}\right) /\left[\alpha\left(r_{t}\right)\right]^{2}\right) \cdot u$.

Now suppose $\Sigma$ is of type $C_{l}(l \geqq 3)$ and $e$ is given by $x_{r_{i}}(t) \rightarrow x_{r_{i}}(t) x_{r_{N}-2 r_{i}}(u t) x_{r_{N}-r_{i}}\left(K u t^{2}\right) \cdot x_{r_{N}}\left(L u t^{3}\right)$-àgain for appropriate $K$ and $L$. Then computing as above we find $d e d^{-1}$ is the extremal automorphism

$$
x_{r_{i}}(t) \rightarrow x_{r_{i}}(t) x_{r \sim-2 r_{i}}\left(u^{\prime} t\right) x_{r N-r_{i}}\left(K u^{\prime} t^{2}\right) x_{r N}\left(L u^{\prime} t^{2}\right)
$$

where $u^{\prime}=\left(\alpha\left(r_{N}\right) / \alpha\left(r_{i}\right)^{3}\right) \cdot u$. Hence, in any case, $D(S)$ normalizes $E(S)$ and hence $W(S)$.

The last assertion of the proposition will follow if we can show that $D \cap \bar{W}=\{1\}$. Let $w \in \bar{W}(S)$ and $d \in D(S)$ corresponding to the character $\alpha$. Write $w=e i c$ with $e \in E(S), \quad c \in C(S)$ and $i \in$ $I(S)$. Then if $d=w$ we have $c=i^{-1} e^{-1} d$. Let $r_{t} \in \Phi$. Then for all $t \in \Gamma\left(S, O_{s}\right)$ we have

$$
\begin{aligned}
c\left[x_{r_{i}}(t)\right]=x_{r_{i}}(t) x_{r N}\left(c_{i}(t)\right)= & i^{-1} e^{-1} d\left[x_{r_{i}}(t)\right] \\
= & i^{-1} e^{-1}\left[x_{r_{i}}\left(\alpha\left(r_{i}\right) t\right)\right] \\
= & x_{r_{i}}\left(\alpha\left(r_{i}\right) t\right) \cdot x_{s}(t) \cdots \\
& \text { with } r_{t}<s .
\end{aligned}
$$


Thus $\alpha\left(r_{\imath}\right)=1$ for all $r_{\imath} \in \Phi$ hence $d=1$.

Let $\mathbf{G}=D \bar{W}$. We call $G$ the connected component of $\mathbf{A}_{V}$. The next proposition justifies this terminology.

Proposition 2.3. The subgroup $\Pi$ normalizes $\mathbf{G}$ and $\mathbf{A}_{V}$ is the semi-direct product of $\Pi$ and $\mathbf{G}$.

Proof. We have seen in 2.1 that $\Pi$ normalizes $\mathbf{C}$ and it clearly normalizes $I$, so it remains to show that $\Pi$ normalizes $D$ and $E$.

Let $d \in D(S)$ correspond to the character $\alpha$. Then if $\rho \in \Pi$, $\rho d \rho^{-1}\left[x_{r}(t)\right]=x_{r}\left(\alpha\left(\rho^{-1}(r)\right) t\right)$. Hence $\Pi$ normalizes $D$.

Now if $r_{t} \in \Phi$ and $r_{N}-r_{t} \in \Sigma^{+}$, then $\rho\left(r_{N}-r_{t}\right)=r_{N}-\rho\left(r_{t}\right) \in \Sigma^{+}$, for $\rho \in \Pi$. Thus $\rho\left(r_{i}\right)=r_{i}$ if $\Sigma$ is not of type $A_{l}$ and $\rho\left(r_{1}\right)=r_{l}$ if $\Sigma$ is of type $A_{l}$ and $r_{N}-r_{i} \in \Sigma^{+}, i=1, l$. A straightforward computation now shows that, if $e \in E(R)$ and $e\left[x_{r_{i}}(t)\right]=x_{r_{i}}(t) x_{r N-r_{i}}(u t) \cdot x_{r_{N}}\left(K u t^{2}\right)$, then $\rho e \rho^{-1}=$ $e^{\prime} \in E(R)$. In fact, if $\Sigma$ is not of type $A_{l}$, then $\rho e \rho^{-1}=e$. If $\Sigma$ is of type $C_{l}(l \geqq 3)$, then $\pi=1$ and there is nothing to prove.

Now suppose $\rho \in \Pi$ and $\rho=\operatorname{diec}, d \in D(S), i \in I(S), e \in E(S)$ and $c \in C(S)$. Then $d^{-1} \rho$ maps $X_{r_{i}}(S)$ onto $X_{\rho\left(r_{i}\right)}(S)$. But iec acts trivially on $V(S) / V_{2}(S)$, since $\Sigma \neq A_{2}$ or $B_{2}$. Hence $\rho\left(r_{1}\right)=r_{1}$ and $\rho$ is trivial. This completes the proof.

We need the following well known lemma.

LEMMA 2.4. Let $v=x_{s_{1}}\left(t_{1}\right) \cdot x_{s_{2}}\left(t_{2}\right) \cdots \cdots x_{s K}\left(t_{K}\right)$ be an element of $V(S)$ with $s_{1}<s_{2}<\cdots<s_{K}$. Then for all $r \in \Sigma^{+}$and all $t \in \Gamma\left(S, O_{S}\right)$

$$
\text { (int } v) x_{r}(t)=x_{r}(t) x_{r+s_{t}}\left(C_{11 r_{s_{t}}}\left(-t_{t} t\right)\right) \cdots
$$

where $i \leqq K$ is the least integer such that $r+s_{i} \in \Sigma^{+}$.

Proof. If $K=1$ the result follows from Chevalley's commutator formula [1: 5.2.2]. The general case follows by a straight forward induction argument.

Proposition 2.5. An element $c \in \mathbf{C}(S)$ lies in $E(S) \cdot I(S)$ if and only if it lies in $I(S)$ and is conjugation by an element of $V_{h-1}(S)$, where $h=h\left(r_{N}\right)$.

Proof. Suppose $c=e \cdot \gamma \neq 1$. Let $r_{t} \in \Phi$ with $r_{N}-r_{t} \notin \Sigma^{+}$. Then

$$
\begin{aligned}
c\left[x_{r_{i}}(t)\right] & =x_{r_{i}}(t) x_{r_{N}}\left(c_{i}(t)\right) \\
& =e \gamma\left[x_{r_{i}}(t)\right] \\
& =x_{r_{i}}(t) x_{r_{i}+s_{i}}\left(N_{r_{s}, s_{j}}\left(-t t_{j}\right) \cdots\right.
\end{aligned}
$$


where $\gamma=\operatorname{int} x_{s_{1}}\left(t_{1}\right) \cdots x_{s_{K}}\left(t_{K}\right)$ and $j$ is the least integer such that $r_{i}+s_{j} \in$ $\Sigma^{+}$. Equating terms, we see that $r_{\imath}+s_{\jmath}=r_{N}$; i.e., $s_{j}=r_{N}-r_{\imath} \in \Sigma^{+}$. This is a contradiction unless $\gamma$ acts trivially on $x_{r_{i}}(t)$ and $c_{i}(t) \equiv 0$. Hence $c\left[x_{r_{i}}(t)\right]=x_{r_{i}}(t)$ unless $r_{N}-r_{i} \in \Sigma^{+}$.

Now suppose $r_{N}-r_{t} \in \Sigma^{+}, r_{t} \in \Phi$ and $\Sigma$ is not of type $C_{l}$.

Then

$$
\begin{aligned}
c\left[x_{r_{i}}(t)\right]= & x_{r_{i}}(t) x_{r_{N}}\left(c_{i}(t)\right) \\
= & e\left[x_{r_{i}}(t) x_{r_{i}+s_{j}}\left(N_{r_{s} j_{j}}\left(-t t_{j}\right)\right) \cdots\right] \\
= & x_{r_{i}}(t) x_{r_{N}-r_{i}}(u t) x_{r_{N}}\left(\frac{1}{2} N_{r N-r_{r} r_{i}} u t^{2}\right) \\
& \cdot x_{r_{i}+s_{j}}\left(N_{r_{s},}\left(-t t_{j}\right)\right) \cdots
\end{aligned}
$$

We must have $r_{t}+s_{j}=r_{N}-r_{i}$. But then $s_{j}=r_{N}-2 r_{t} \in \Sigma^{+}$a contradiction, thus we conclude $e=1$.

If $\Sigma$ is of type $C_{l}$, then we have

$$
\begin{aligned}
c\left[x_{r_{i}}(t)\right]= & x_{r_{i}}(t) x_{r N}\left(c_{i}(t)\right) \\
= & e\left[x_{r_{i}}(t) x_{r_{i}+s_{j}}\left(N_{r_{i j}}\left(-t t_{j}\right)\right) \cdots\right. \\
= & x_{r_{i}}(t) x_{r N-2 r_{i}}(f(t)) x_{r N-r_{i}}(g(t)) x_{r N}(h(t)) \\
& \cdot x_{r_{i}+s_{j}}\left(N_{r_{i} s_{j}}\left(-t t_{j}\right)\right) \cdots .
\end{aligned}
$$

Now if $f$ is not identically zero, we have $r_{t}+s_{l}=r_{N}-2 r_{t}$ so that $s_{j}=r_{N}-3 r_{\imath} \in \Sigma^{+}$. But this is impossible since the Cartan matrix for $C_{l}$ shows that the $r_{i}$ chain of roots through $r_{N}$ has length 2. Thus $f$ is identically zero. It must happen, then, that $r_{t}+s_{\jmath}=r_{N}-r_{t}$ so that $s_{J}=r_{N}-2 r_{1}$ and $g(t)=N_{r, r_{N}-2 r_{i}}\left(t t_{j}\right)$.

We have shown that $e$ is given by

$$
x_{r_{i}}(t) \rightarrow x_{r_{i}}(t) x_{r_{N}-r_{i}}(u t) x_{r_{N}}\left(\frac{1}{2} N_{r N-r_{i} r_{i}} u t^{2}\right)
$$

where $\quad u=N_{r, r_{N}-2 r_{i}} t_{j}=-N_{r_{N}-2 r_{,}, r_{i}} t_{j} . \quad\left(\right.$ Recall $\quad N_{r s}=-N_{s r} \quad$ all $\quad r, s \in$ $\Sigma^{+}$.) Consider now the effect of int $x_{r-2 r_{i}}\left(t_{j}\right)$. Recall $r_{N}-2 r_{i}+r \in \Sigma^{+}$ only if $r=r_{i}$. Thus we need only consider int $x_{r_{N}-2 r_{i}}\left(t_{l}\right)$ acting on $x_{r_{i}}(t)$ :

$$
\text { int } \begin{aligned}
x_{r_{N}-2 r_{r}}\left(t_{j}\right)\left[x_{r_{i}}(t)\right]= & x_{r_{i}}(t) x_{r_{N}-r_{i}}\left(N_{r_{r}, r_{N}-2 r_{i}}\left(-t t_{j}\right)\right) \\
& \cdot x_{r_{N}}\left(C_{21, r_{i}, r_{N}-2 r_{i}} t^{2} t_{l}\right) \\
= & x_{r_{i}}(t) x_{r_{N}-r_{l}}(-u t) x_{r_{N}}\left(C_{21, r_{r}, r_{N}-2 r_{i}} t_{j} t^{2}\right) .
\end{aligned}
$$

But

$$
\begin{aligned}
C_{21, r_{i}, r_{N}-2 r_{r} t_{j}} & =\frac{1}{2} N_{r_{r}, r_{N}-2 r_{i}} N_{r_{r}, r_{N}-r_{r}} t_{j} \\
& =\frac{1}{2} N_{r_{r}, r_{N}-r_{1}} u=-\frac{1}{2} N_{r N-r_{r}, r_{i}} u .
\end{aligned}
$$


Thus int $x_{r_{N}-2 r_{i}}\left(t_{j}\right)=e^{-1}$ and, hence, if $c \in C(S) \cap E(S) I(S)$, then $c \in$ $I(S)$.

It follows that for $r_{i} \in \Phi, r_{N}-r_{i} \in \Sigma^{+}$

$$
\begin{aligned}
c\left[x_{r_{i}}(t)\right] & =x_{r_{l}}(t) x_{r N}\left(c_{l}(t)\right) \\
& =x_{r_{i}}(t) x_{r_{i}+s_{j}}\left(N_{r_{i s}}\left(-t t_{j}\right)\right) \cdots .
\end{aligned}
$$

Equating terms we see that $r_{t}+s_{j}=r_{N}$ so $s_{j}=r_{N}-r_{i}$. If $\Sigma$ is not of type $A_{l}$, then we could only have $\gamma=$ int $x_{r_{N}-r_{i}}(\alpha)$, since there is just one root of height $h-1$. In this case, we have

$$
\text { int } x_{r_{N}-r_{i}}(\alpha)\left[x_{r_{i}}(t)\right]=x_{r_{i}}(t) x_{r_{N}}\left(N_{r_{,}, r_{N}-r_{i}}(-\alpha t)\right) \text {. }
$$

Again equating terms we see $N_{r_{N}-r_{r}, r_{1}} \alpha t=c_{i}(t)$.

If $\Sigma$ is of type $A_{l}$, then there are two roots $r_{1}, r_{l} \in \Phi$ with $r_{N}-r_{l} \in$ $\Sigma^{+}$. Just as above, we see that conjugation by a suitable element of $X_{r_{N} r_{i}}(S), i=1, l$, has the same effect as a central automorphism on $X_{r_{i}}(S)$, $i=1, l$. In particular, these two types of automorphisms commute with each other since $\Sigma$ is not of type $A_{2}$ and thus their product gives an element of $C(S)$. Since the $x_{r_{i}}(t), r_{t} \in \Phi, t \in \Gamma\left(S, O_{s}\right)$ generate $V(S)$ as a group, the proof is complete.

\section{Proposition 2.6. The subfunctor $W=E I$ is representable.}

Proof. We shall show that $W$ is the semi-direct product of two representable functors. Suppose first that $\Sigma$ is not of type $C_{l}$. We claim that, in this case, $W$ is the semi-direct product of $E$ and $I$.

Let $e \in E(S) \cap I(S)$, say $e=\gamma$ with $\gamma=$ int $x_{s_{1}}\left(t_{1}\right) \cdots x_{s_{K}}\left(t_{K}\right)$. Then if $r_{N}-r_{i} \in \Sigma^{+}, r_{i} \in \Phi$, using Lemma 2.4 we have

$$
\begin{aligned}
e\left[x_{r_{i}}(t)\right] & =x_{r_{i}}(t) x_{r_{N}-r_{i}}(u t) x_{r_{N}}\left(\frac{1}{2} N_{r_{N}-r_{r_{i}} r_{i}} u t^{2}\right) \\
& =\gamma\left[x_{r_{i}}(t)\right]=x_{r_{i}}(t) x_{r_{i}+s_{1}}\left(N_{r_{i}, r_{i}+s_{i}}-t_{1} t\right) \cdots .
\end{aligned}
$$

Equating terms we see that $r_{i}+s_{j}=r_{N}-r_{i}$; hence $s_{J}=r_{N}-2 r_{i} \in \Sigma^{+}$, a contradiction. Hence $e=\gamma=1$.

Now suppose $\Sigma$ is of type $C_{l}$. Then

$$
\begin{aligned}
e\left[x_{r_{i}}(t)\right] & =x_{r_{i}}(t) x_{r_{N}-2 r_{i}}(u t) \cdots=\gamma\left[x_{r_{i}}(t)\right] \\
& =x_{r_{i}}(t) x_{r_{i}+s_{j}}\left(N_{r_{r} r_{i}+s_{j}}\left(t t_{j}\right) \cdots\right.
\end{aligned}
$$

Thus $r_{\imath}+s_{j}=r_{N}-2 r_{t}$ so $s_{j}=r_{N}-3 r_{\imath} \in \Sigma^{+}$, a contradiction. Hence $e$ must be of the type considered in the first part of the proof. Equating 
terms again, we find that $s_{J}=r_{N}-2 r_{i}$. As in the proof of Proposition 2.5, we can find $\alpha \in \Gamma\left(S, O_{S}\right)$ such that int $x_{r_{N}-2 r_{r}}(\alpha)=e$. Now put

$$
\begin{aligned}
E_{1}=\left\{e \in E(S) \mid e\left[x_{r_{i}}(t)\right]\right. & =x_{r_{i}}(t) x_{r_{N}-2 r_{i}}(u t) \cdots, \\
e\left[x_{r}(t)\right] & \left.=x_{r}(t), r \neq r_{l}\right\} .
\end{aligned}
$$

Then $E_{1} \cap I(S)=1$ so $W$ is the semi-direct product of $E_{1}$ and $I(S)$.

Finally, since $E, E_{1}$ and $I$ are representable, so is $W$.

Let $S$ be a reduced $A$-algebra and $c$ an element of $\Lambda(S)=$ Hom $_{s-g r}\left(G_{a} \times{ }_{A} S, G_{a} \times{ }_{A} S\right)$. Then, since $G_{a} \times{ }_{A} S=\operatorname{Spec} O_{S}[T]$, the element $c$ is completely determined by a polynomial $c(T)$ in $O_{S}[T]$. We can write $c(T)=c_{1}(T)+c_{2}(T)$, where $c_{1}(T)$ has degree $\leqq 1$ and $c_{2}(T)$ contains no terms of degree less than 2 . It follows that $\mathbf{C}(S)=$ $C_{1}(S) \times C_{2}(S)$, where $C_{1}(S)=\left\{c \in C(S)\right.$ : degree $\left.c_{1} \leqq 1\right\}$ and $C_{2}(S)=$ $\{c \in C(S): c$ has no terms of degree less than 2$\}$. It is also clear that if $c \in C_{1}(S)$, then $c$ has no constant term. Thus $C=C_{1} \times C_{2}$ where $C_{1} \simeq G_{a .}^{l}$. An entirely straight forward computation shows that $C_{1}$ and $C_{2}$ are both normal subfunctors of $\mathbf{A}_{V}$. In fact, $E$ and $I$ centralize $C$ so one need only check conjugation by elements of $D$ and $\pi$ and the result for these subgroups follows essentially from the definitions.

We define $H$ to be the subfunctor of $\mathbf{A}_{V}$ generated by $\pi, D, E, I$ and $C_{1}$ and set $N=C_{2}$.

Proposition 2.7. For any A-scheme $S, \mathbf{A}_{V}(S)$ is the semi-direct product of $H(S)$ and $N(S)$.

Proof. Clearly $\langle H(S), N(S)\rangle=\mathbf{A}_{V}(S)$ and it suffices to show that $H(S) \cap N(S)=1$. This follows from 2.2, 2.3 and 2.5.

COROLlaRY 2.8. The subgroup $H$ is represented by a smooth solvable A-group scheme and $\mathbf{A}_{V}$ is a sheaf on the Zariski site $\mathrm{Sch} / A$.

Proof. Let $H_{0}=\left\langle D, E, I, C_{1}\right\rangle$. Then

$$
1 \rightarrow H_{0} \rightarrow H \rightarrow \pi \rightarrow 1
$$

is exact. But $\pi$ is solvable by 1.4 and the solvability of $H_{0}$ follows from 2.2 and 2.5. That $H$ is representable is a consequence of the semi-direct product decompositions $H=\pi \cdot H_{0}, H_{0}=D \cdot E I C_{1}$, Proposition 2.6 and the representability of $D, E, I, C_{1}$ and $\pi$.

The second assertion will follow if we show $N$ is a sheaf since $\mathbf{A}_{V}=H \cdot N$. But $N \cong \prod_{n=1}^{\infty} G_{a}$ which is clearly a a sheaf on $\operatorname{Sch} / A$. 
THEOREM 2.8. Let $S$ be a reduced A-scheme. Then the canonical map $j: \mathbf{A}_{V}(S) \rightarrow \operatorname{Aut}_{V}(S)$ is an isomorphism. Consequently $\mathbf{A}_{V} \simeq A u t_{V}$ on $(\mathrm{Sch} / A)_{\mathrm{red}}$.

Proof. Let $S \in(\mathrm{Sch} / A)_{\text {red }}$ be given and $\left\{S_{t}\right\}$ an open affine covering of $S$. Since $\mathbf{A}_{V}$ and $A u t_{V}$ are sheaves we have the following commutative diagram

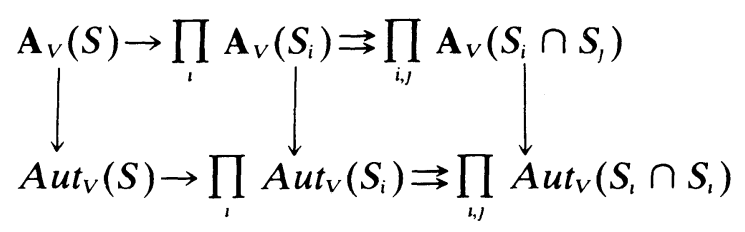

where the rows are exact. It follows that the theorem holds if and only if it holds for affine schemes $S$. Moreover, since connected components are open, we may also assume each $S_{t}$ is also connected so it is enough to establish the result when $S=\operatorname{Spec} R$ is reduced and connected.

The theorem holds when $R$ is a field by [4: Theorem 6.2]. We indicate now how each step used in the proof of the case for fields can be carried out over $R$. The proof proceeds through seven steps. We write $A u t_{V}(R)$ for $A u t_{V}(\operatorname{Spec} R)$.

I. Let $t_{1}, \cdots, t_{l}$ be arbitrary nonzero elements of $R$ and suppose $\theta \in A u t_{v}(R)$. If $\theta\left[x_{r_{i}}\left(t_{t}\right)\right]=\prod_{j=1}^{l} x_{r_{1}}\left(t_{t l}\right) \bmod V_{2}(R)$, then the matrix $T=$ $\left[t_{1}\right]$ is monomial; i.e., $T$ has just one entry in each row and each column. Consequently, $\theta\left(X_{r_{1}}\right)=X_{r_{p(t)}}\left(\bmod V_{2}\right)$, where $\rho$ is some permutation of $1, \cdots, l$.

Proof. Let $y_{1}, \cdots, y_{l}$ be indeterminants and put $A^{\prime}=$ $R\left[y_{1}, \cdots, y_{l}\right]$. Let $\theta_{A^{\prime}}$ be the image of $\theta$ under the homomorphism $\operatorname{Aut}_{V}(R) \rightarrow \operatorname{Aut}_{V}\left(A^{\prime}\right)$. We confuse $\theta_{A^{\prime}}$ with $\theta_{A^{\prime}}\left(A^{\prime}\right) \in$ Aut $_{\mathrm{Gr}}(V(A))$. Suppose $\theta_{A^{\prime}}\left(x_{r_{i}}\left(y_{i}\right)\right) \equiv \prod_{j=1}^{l} x_{r_{i}}\left(y_{i j}\right) \bmod V_{2}\left(A^{\prime}\right)$ with $y_{1,} \in A^{\prime}, 1 \leqq i \leqq l$. Let $p \in \operatorname{Spec} R$. Then $\bar{y}_{1} \neq 0$ in $R / p\left[y_{1}, \cdots, y_{l}\right]=$ $A^{\prime} / p$ and we have $\theta_{A^{\prime} / p}\left[\bar{y}_{i}\right] \equiv \prod_{j=1}^{l} x_{r_{j}}\left(\bar{y}_{i j}\right)$. The automorphism $\theta_{A^{\prime} / P}$ extends uniquely to an automorphism of $V(K)$ where $K$ is the quotient field of $A / P$. Then by $[4: 6.3]$ the matrix $T_{A / P}=\left[\bar{y}_{i j}\right]$ is monomial. Since this holds for all $p \in \operatorname{Spec} R, R$ is reduced and $S$ is connected we have shown that $T_{A^{\prime}}=\left[y_{i j}\right]$ is monomial.

Now let $\varphi: A^{\prime} \rightarrow R$ be the $R$-algebra homomorphism determined by $\varphi\left(Y_{i}\right)=t_{t}, \quad 1 \leqq i \leqq l$. This induces a group homomorphism $\bar{\varphi}: V\left(A^{\prime}\right) \rightarrow V(R)$ and moreover

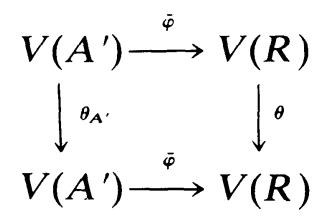


commutes. It follows that $T=\left[t_{i j}\right]=\varphi\left[T_{A^{\prime}}\right]=\left[\varphi\left(y_{i j}\right)\right]$ is monomial and that $\theta\left[X_{r_{i}}\right] \equiv X_{r_{\rho(1)}} \bmod V_{2}$ for some permutation $\rho$ of $1,2, \cdots, l$.

II. Let $\theta \in A u t_{V}(R)$. Then there exist a graph automorphism $g \in \Pi$ such that $g^{-1} \theta\left(X_{r_{i}}(R)\right) \equiv X_{r_{i}}(R) \bmod V_{2}(R)$ for each fundamental root subgroup $X_{r_{i}}(R), r_{i} \in \Phi$.

Proof. By step I, $\theta\left(X_{r_{i}}(R)\right) \equiv X_{r_{o(t)}}(R) \bmod V_{2}(R)$ for $1 \leqq i \leqq l$, where $\rho$ is some permutation of $1,2, \cdots, l$. Then it follows as in [4:6.4] that $\rho$ induces a symmetry of $\Sigma$ - the set of all roots. The corresponding graph automorphism $g \in \Pi$ does the trick.

III. Let $g$ and $\theta$ be as in II. Then there is a diagonal automorphism $d \in D(R)$ such that $d^{-1} g^{-1} \theta$ acts trivially on $V(R) \bmod V_{2}(R)$.

Proof. By II, $g^{-1} \theta$ induces an automorphism of $V(R) / V_{2}(R)$ which sends $X_{r_{i}}(R) \bmod V_{2}(R)$ into itself. Let $g^{-1} \theta\left[x_{r_{i}}(1)\right] \equiv x_{r_{i}}\left(u_{i}\right) \bmod$ $V_{2}(R)$. Then $g^{-1} \theta$ acts on $V(R) / V_{2}(R) \cong A^{l}(R)$ via the matrix $T=$ $\operatorname{diag}\left(u_{1}, \cdots, u_{l}\right)$. Since $g^{-1} \theta$ is an automorphism, it follows that $u_{l}$ is a unit in $R$ all $i$. Let $d \in \mathbf{D}(R)$ be the diagonal automorphism determined by the homomorphism $\alpha: M \rightarrow R^{*}$ given by $\alpha\left(r_{\imath}\right)=u_{i}, 1 \leqq i \leqq l$. Then $d^{-1} g^{-1} \theta$ acts trivially on $V(R) / V_{2}(R)$.

IV. Let $\theta \in A u t_{V}(R)$ and suppose $\theta$ acts trivially on $V \bmod$ $V_{2}$. Then there is an inner automorphism $i \in I$ such that $i^{-1} \theta$ acts trivially on $V \bmod V_{m}$ where $m=h-1$ if $\Sigma$ is not of type $C_{l}$ and $m=h-2$ if $\Sigma$ is of type $C_{l}$ and where $h=h\left(r_{N}\right)$ is the height of the highest root.

Proof. It clearly suffices to show that if $2 \leqq n \leqq m-1$ and $\theta$ acts trivially on $V \bmod V_{n}$, then there exist an inner automorphism $i \in I$ such that $i^{-1} \theta$ acts trivially on $V \bmod V_{n+1}$.

Let $s \in \Sigma^{+}$have height $n$ and suppose that for some fundamental root $r_{i} \in \Phi$

$$
\theta\left[x_{r_{i}}(t)\right]=x_{r_{i}}(t) x_{s}(f(t)) \cdots
$$

all $t \in R$ with $f \in R[x]$ not identically zero. Then by arguments similar to those in [4:6.7], $s-r_{t} \in \Sigma^{+}$. For the remainder of the argument we replace $s-r_{i}$ by $s$ so $h(s)=n-1$ and

$$
\theta\left[x_{r_{i}}(t)\right]=x_{r_{i}}(t) x_{s+r_{i}}(f(t)) .
$$

We claim there is an element $i_{\mathrm{s}} \in I$ such that the element $i_{s}=$ 
$i_{s}(R) \in I(R)$ acts trivially on $V(R) \bmod V_{n}(R)$ and $i_{s}\left[x_{r_{i}}(1)\right]=$ $x_{r_{i}}(1) x_{s+r_{i}}(f(1)) \cdots$.

To see this, suppose $i_{s}=$ int $x_{s}(\alpha)$ with $\alpha \in R$ to be determined. Then $i_{s}$ acts trivially on $V(R) \bmod V_{n}(R)$ by the Chevalley commutator formula [1:5.2.2]. Moreover

$$
x_{s}(-\alpha) x_{r_{i}}(1) x_{s}(\alpha)=x_{r_{i}}(1) x_{s+r_{i}}\left(C_{11, s, r_{i}}-\alpha \cdot 1\right) \cdots \text {. }
$$

Since $C_{11, s, r_{i}} \neq 0$ is an integer whose absolute value is less than $4, C_{11, s, r_{i}}$ is a unit in $R$ and we take $\alpha= \pm C_{11, s, r}^{-1} f(1)$.

We now have $i_{s}^{-1} \theta\left[x_{r_{i}}(t)\right]=x_{r_{i}}(t) x_{s+r_{i}}(g(t)) \cdots$ where $g(1)=0$. We claim that $g$ is identically zero and moreover if there is an $r_{j} \in \Phi, i \neq j$, such that $s+r_{j} \in \Sigma^{+}$and $i_{s}^{-1} \theta\left[x_{r_{j}}(t)\right]=x_{r_{j}}(t) x_{s+r_{j}}\left(g^{\prime}(t)\right) \cdots$, then $g^{\prime}$ is also identically zero. But all of the above relations hold after reduction to $R / P$ for any $p \in \operatorname{Spec} R$. If $K$ is the quotient field of $R / P$, the above relations hold for the image of $i_{s}^{-1} \theta$ in $\operatorname{Aut}_{G r}(V(K))$. Then by arguments similar to those given in [4:6.7] $g$ and $g^{\prime}$ are identically zero.

Now we can find such an inner automorphism $i_{s}$ for each root $s$ such that $h(s)=n-1$. If we put $i$ equal to the product of all these inner automorphisms, then $i^{-1} \theta$ acts trivially on $V(R) / V_{n+1}(R)$ and the lemma is proved.

The last three stages of the argument consist in showing the following:

V. If $V$ is of type $C_{l}$ and $\theta \in A u t_{V}(R)$ acts trivially on $V(R) \bmod$ $V_{h-2}(R)$ then there are extremal and inner automorphisms $i$ and $e$ in $A u t_{V}(R)$ such that $e^{-1} i^{-1} \theta$ acts trivially on $V(R) \bmod V_{h-1}(R)$.

VI. If $\theta \in A u t_{V}(R)$ acts trivially on $V(R) \bmod V_{h-1}$ then there exist an inner automorphism $i \in I(R)$ such that $i^{-1} \theta$ acts trivially on $V(R) \bmod V_{h-1}(R)$ and on $V_{2}(R)$.

VII. If $\theta \in A u t_{V}(R)$ acts trivially on $V(R) \bmod V_{h-1}(R)$ and on $V_{2}(R)$ then $\theta=i e c$ where $i \in I(R), e \in E(R)$ and $c \in \mathbf{C}(R)$.

The proofs of these assertions follow from adaptations of the proofs of Lemmas 6.8, 6.9 and 6.10 of [4] similar to the arguments we have given above.

We have shown that the subfunctors $\Pi, D ; I, E$ and $\mathbf{C}$ generate $A u t_{V}$ when these functors are restricted to $(\mathrm{Sch} / A)_{\text {red }}$ and $V$ is not of type $B_{2}$ or $A_{2}$. These two special groups can be treated directly just as in [4:6.11]. We discuss them in $\$ 3$. 
Now let $k$ be a field whose characteristic is different from 2 and 3. Applying the base change functor $-x_{A} k$ we obtain $V_{k}$ a unipotent $k$-group of Chevalley type. In this case we can summarize the above results as follows:

Corollary 2.9. Let $V=V(\Phi, \Sigma)$ be a unipotent $k$-group of Chevalley type. Assume that char $k \neq 2,3$ and that $\Sigma$ is not of type $A_{2}$ or $B_{2}$. Let $A u t_{V}(S)=\operatorname{Aut}_{s-g r}\left(V \times_{k} S\right)$ for all $S$ in $(\mathrm{Sch} / k)_{\text {red. }}$ Then

(i) There exists an exact sequence of group valued functors on $(\mathrm{Sch} / k)_{\text {red }}$

$$
1 \rightarrow N \rightarrow A u t_{V} \rightarrow H \rightarrow 1
$$

making $A t_{V}$ the semi-direct product of $\mathbf{H}$ and $N$.

(ii) The functor $H$ is representable by a smooth solvable algebraic $k$-group scheme.

(iii) If char $k \neq 0$ then $N \simeq \coprod_{n=1}^{\infty} G_{a}$.

(iv) If char $k=0$ then $N=0$ and $A u t_{V} \simeq H$.

3. The cases $\Sigma=A_{2}$ or $B_{2}$. Let $\Sigma=A_{2}, V=V(\Phi, \Sigma)$ and $\mathbf{A}_{V}$ be the subgroup of $A u t_{V}$ generated by $\Pi, D, E, I$ and $C$. Let $\Sigma=\{r, s, r+s\}$. An easy computation shows that $I \subset C$ in this case.

THEOREM 3.1. Let $S$ be an A-scheme and $\rho: \mathbf{A}_{V}(S) \rightarrow$ Aut $_{s-g r}$ $\left(V / X_{r+s} \times_{A} S\right)$ be the canonical homomorphism induced by passage to the quotient. Then the image of $\rho$ is isomorphic to $\mathrm{GL}_{2}(S)$, the kernel of $\rho$ is $\mathrm{C}(S)$ and the exact sequence

$$
1 \rightarrow \mathbf{C}(S) \rightarrow \mathbf{A}_{V}(S) \rightarrow \mathrm{GL}_{2}(S) \rightarrow 1
$$

has a section making $\mathbf{A}_{V}(S)$ the semi-direct product of $\mathrm{GL}_{2}(S)$ and $\mathbf{C}(S)$. In particular $\mathbf{A}_{V} \simeq \mathrm{GL}_{2} \cdot C$.

Proof. Let $\theta \in \mathbf{A}_{V}(S)$ and suppose $\theta$ acts trivially on $V / X_{r+s} \times_{A} S$. Then $\theta\left(x_{r}(t)\right)=x_{r}(t) x_{r+s}\left(f_{r}(t)\right)$ and $\theta\left(x_{s}(t)\right)=x_{s}(t) x_{r+s}\left(f_{s}(t)\right)$ for all $t \in \Gamma\left(S, O_{s}\right)$. It is easy to see that $f_{r}$ and $f_{s}$ give rise to a unique $c \in \mathbf{C}(S)$ and that $\theta=c$. This shows that $C(S)$ is the kernel of $\rho$.

Since $\mathrm{A}_{V}(S)$ is generated by $\Pi, D(S), E(S)$ and $C(S)$, and since $I(S) \subset C(S)=\operatorname{Ker} \rho$, to show that $\operatorname{Im} \rho=\mathrm{GL}_{2}(S)$ it suffices to show that the images of $\Pi, D(S)$ and $E(S)$ under $\rho$ lie in $\mathrm{GL}_{2}(S)$. For $\Pi$ and $D(S)$ this is clear. Recall that $E(S)$ is generated by two types of extremal automorphism $e_{1}$ and $e_{2}$ : $e_{1}$ fixes $X_{s}(S)$ and $X_{r+s}(S)$ and maps $x_{r}(t)$ to $x_{r}(t) x_{s}(a t) x_{r+s}\left(\frac{1}{2} a t^{2}\right), \quad a, t \in G_{a}(S)$ and $e_{2}=\sigma \cdot e_{1} \sigma$ where $\sigma$ is the generator of $\Pi$. Then $\rho\left(e_{1}\right)$ is represented by the matrix $\left[\begin{array}{ll}1 & a \\ 0 & 1\end{array}\right]$ in $\operatorname{GL}_{2}(S)$ 
and it follows that $\rho(E(S)) \subset \mathrm{GL}_{2}(S)$ so $\operatorname{Im} \rho=\mathrm{GL}_{2}(S)$. For any matrix $M=\left|\begin{array}{ll}a & b \\ c & d\end{array}\right|$ in $\mathrm{GL}_{2}(S)$ define $S^{\prime}(M): V(S) \rightarrow V(S)$ as follows

$$
\begin{aligned}
& x_{r}(t) \rightarrow x_{r}(a t) x_{s}(b t) x_{r+s}\left(-a b t^{2}\right) \\
S^{\prime}(M): \quad & x_{s}(t) \rightarrow x_{r}(c t) x_{s}(d t) x_{r+s}\left(\frac{1}{2} c d t^{2}\right) \\
& x_{r+s}(t) \rightarrow x_{r+s}((a d-b c) t) .
\end{aligned}
$$

It is an easy matter to verify that $S^{\prime}(M)$ induces an automorphism of $V(S)$ hence also $V x_{A} S$. It follows that $\rho$ is surjective and as one sees easily $S^{\prime}$ is a homomorphism so that the remaining assertions of the theorem follow.

COROllary 3.2. The group valued functors $\mathbf{A}_{V}$ and $A u t_{V}$ are isomorphic on $(\mathrm{Sch} / A)_{\mathrm{red}}$.

Proof. The corollary follows from a straight forward adaptation of Gibbs result [4:6.11] similar to the proof of Theorem 2.9 using the fact that $\mathbf{A}_{V} \simeq \mathrm{GL}_{2} \cdot C$ is a sheaf.

Corollary 3.3. Let $\Sigma=A_{2}$ and $k$ be a field with char $k \neq 2,3$. Let $V=V(\Phi, \Sigma)_{k}$ and Aut $t_{V}$ the functor on $(\mathrm{Sch} / k)_{\text {red }}$ given by $S \rightarrow$ Aut $_{s-g r}\left(V \times{ }_{k} S\right)$. Then there exists an exact sequence of group valued functors

$$
1 \rightarrow N \rightarrow A u t_{V} \rightarrow H \rightarrow 1
$$

on $(\mathrm{Sch} / k)_{\mathrm{red}}$ such that

(i) $H$ is representable by an affine algebraic $k$-group.

(ii) $A u t_{v} \simeq H \cdot N$.

(iii) There is a split exact sequence

$$
1 \rightarrow I \rightarrow H \rightarrow \mathrm{GL}_{2} \rightarrow 1
$$

(iv) If char $k \neq 0, N \simeq \amalg_{n=1}^{\infty} G_{a}$ and if char $k=0, N=0$.

Proof. Everything has been established except (iii) which follows from the fact that (using the notation of 2.7) $E_{1}=I$.

When $\Sigma=B_{2}, \Pi=1$ and $\mathbf{A}_{V}$ is generated by $D, E, I$ and $C$. By methods entirely analogous to those above, we obtain the following result:

THEOREM 3.4. Let $V=V(\Phi, \Sigma)$ be a unipotent A-group of Cheval- 
ley type with $\Sigma=B_{2}$. Then there exists an exact sequence of group valued functors on $(\mathrm{Sch} / A)_{\mathrm{red}}$.

$$
1 \rightarrow N \rightarrow A u t_{V} \rightarrow H \rightarrow 1
$$

such that

(i) $\quad A u t_{V}=H \cdot N$ (semi-direct product).

(ii) $H$ is representable by a connected solvable affine $k$-group. The analogous results to 3.3 hold for $k$ a field, char $k \neq 2,3$.

Remarks. 1. At present we do not know whether the group functors $\mathbf{A}_{V}$ and $\boldsymbol{A u t}_{V}$ are isomorphic as functors on $\mathrm{Sch} / \boldsymbol{A}$. Even over an algebraically closed field of characteristic zero the situation is not clear.

We have excluded the trivial case $V=G_{a}$ throughout. However, in this case, we can see that $\mathbf{A}_{V}$ and $A u t_{V}$ are distinct on $S \mathrm{ch} / k$. In fact, $\mathbf{A}_{V} \simeq G_{m}$ in any characteristic. On the other hand, if $R$ is a nonreduced $k$-algebra and $u \in R, u \neq 0, u^{2}=0$, then the map $R[x] \rightarrow R[x]$ determined by $x \rightarrow x+u \cdot f(x), f(x)$ any additive polynomial gives an automorphism of $G_{a}$ which is not necessarily in $\mathbf{A}_{V}(R)$.

2. If $k$ is a field with char $k \neq 2,3$, then using Gibbs results one obtains immediately that the automorphism group of $V(k)$ (considered now as the $k$-rational points of $V(\bar{k}), \bar{k}$ an algebraic closure of $k$ ) is the semi-direct product of the group $A u t_{V}(k)$ and $\operatorname{Aut}(k)$. In particular, if char $k=0$, one obtains the expected result that $\operatorname{Aut}(V(k))$ is the semi-direct product of a group of matrices over $k$ and the group of automorphisms of the field $k$.

3. Again, let $k$ be a field with appropriate characteristic restrictions. Then it follows from what we have developed above that any finite set of automorphisms of $V \times_{A} k$ is contained in a subgroup of $\operatorname{Aut}_{V}(k)$ which is represented by an algebraic $k$-group. This follows from an examination of the 'central components' of the given automorphisms - for if $\alpha \in N(k)$ then the degrees of the polynomials in $k[x]$ (c.f. $2.6 \mathrm{ff}$ ) determining $\alpha$ are bounded. This bound determines a certain representable subfunctor.

\section{REFERENCES}

1. R. W. Carter, Simple Groups of Lie Type, Wiley, New York, 1972.

2. M. Demazure and A. Grothendieck, (SGA3), Schemas en Groups, Vols. I, II and III, Springer Lecture Notes, Nos. 151, 152, 153. New York, 1970. 
3. A. Fauntleroy, Defining normal subgroups of unipotent algebraic groups, Proc. Amer. Math. Soc., 50 (1975).

4. J. Gibbs, Automorphisms of certain unipotent groups, J. Algebra, 14 (1970).

5. G. P. Hochschild, and G. Mostow, Automorphisms of affine algebraic groups, J. Algebra, 13 (1969).

6. H. Matsumura, Commutative Algebra, Benjamin, New York, 1970.

7. H. Matsumura, and F. Oort, Representability of group functors and automorphisms of algebraic schemes, Inventiones, 4 (1967).

8. J. Sullivan, Automorphisms of affine unipotent groups in positive characteristic, J. Algebra, 26 (1973).

Received March 31, 1976.

UNIVERSITY OF ILLINOIS - URBANA - CHAMPAIGN 


\section{PACIFIC JOURNAL OF MATHEMATICS}

\section{EDITORS}

RICHARI) ARENS (Managing Editor)

University of California

Los Angeles, CA 90024

R. A. Beaumiont

University of Washington

Seattle, WA 98105

\section{J. DugunduI}

Department of Mathematics University of Southern California Los Angeles, CA 90007

D. Gilbarg and J. Milgram Stanford University

Stanford, CA 94305

\section{ASSOCIATE EDITORS}

E. F. BECKENBACH
B. H. NEUMANN

F. Wolf

K. YoshidA

\section{SUPPORTING INSTITUTIONS}

UNIVERSITY OF BRITISH COLUMBIA CALIFORNIA INSTITUTE OF TECHNOLOGY

UNIVERSITY OF CALIFORNIA

MONTANA STATE UNIVERSITY

UNIVERSITY OF NEVADA

NEW MEXICO STATE UNIVERSITY

OREGON STATE UNIVERSITY

UNIVERSITY OF OREGON

OSAKA UNIVERSITY

\author{
UNIVERSITY OF SOUTHERN CALIFORNIA \\ STANFORD UNIVERSITY \\ UNIVERSITY OF HAWAII \\ UNIVERSITY OF TOKYO \\ UNIVERSITY OF UTAH \\ WASHINGTON STATE UNIVERSITY \\ UNIVERSITY OF WASHINGTON \\ AMERICAN MATHEMATICAL SOCIETY
}

The Supporting Institutions listed above contribute to the cost of publication of this Journal, but they are not owners or publishers and have no responsibility for its contents or policies.

Mathematical papers intended for publication in the Pacific Journal of Mathematics should be in typed form or offset-reproduced (not dittoed), double spaced with large margins. Underline Greek letters in red, German in green, and script in blue. The first $p: 1<$ graph or two must be capable of being used separately as a synopsis of the entire paper. Items of the biblography should not be cited there unless absolutely necessary, in which case they must he identified by author and Journal, rather than by item number. Manuscripts, in duplicate, may be sent to any one of the four editors. Please classify according to the scheme of Math. Reviews, Index to Vol. 39. All other communications should be addressed to the managing editor, or Elaine Barth, University of California, Los Angeles, California, 90024.

100 reprints are provided free for each article, only if page charges have been substantially paid. Additional copies may be obtained at cost in multiples of 50 .

The Pacific Journal of Mathematics is issued monthly as of January 1966. Regular subscription rate: $\$ 72.00$ a year (6 Vols., 12 issues). Special rate: $\$ 36.00$ a year to individual members of supporting institutions.

Subscriptions, orders for back numbers, and changes of address should be sent to Pacific Journal of Mathematics, 103 Highland Boulevard, Berkeley, California, 94708.

PUBLISHED BY PACIFIC JOURNAL OF MATHEMATICS, A NON-PROFIT CORPORATION Printed at Jerusalem Academic Press, POB 2390, Jerusalem, Israel.

\section{Copyright (C) 1976 Pacific Journal of Mathematics} All Rights Reserved 


\section{Pacific Journal of Mathematics}

\section{Vol. 66, No. 2 December, 1976}

Gerald A. Beer, Tax structures whose progressivity is inflation neutral..... 305

William M. Cornette, A generalization of the unit interval............. 313

David E. Evans, Unbounded completely positive linear maps on

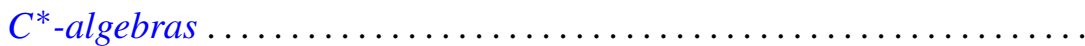

Hector O. Fattorini, Some remarks on convolution equations for

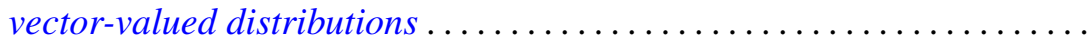

Amassa Courtney Fauntleroy, Automorphism groups of unipotent groups of

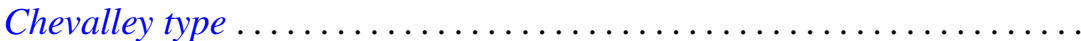

Christian C. Fenske and Heinz-Otto Peitgen, On fixed points of zero index in

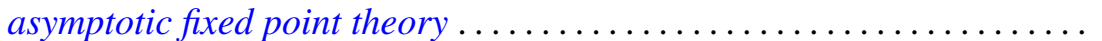

Atsushi Inoue, On a class of unbounded operator algebras. II ............

Herbert Meyer Kamowitz, The spectra of endomorphisms of algebras of

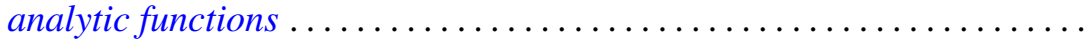

Jimmie Don Lawson, Embeddings of compact convex sets and locally compact cones ....................................

William Lindgren and Peter Joseph Nyikos, Spaces with bases satisfying certain order and intersection properties .....................

Emily Mann Peck, Lattice projections on continuous function spaces ...... 477

Morris Marden and Peter A. McCoy, Level sets of polynomials in $n$ real variables...

Francis Joseph Narcowich, An imbedding theorem for indeterminate Hermitian moment sequences......................

John Dacey O'Neill, Rings whose additive subgroups are subrings ...

Chull Park and David Lee Skoug, Wiener integrals over the sets bounded by sectionally continuous barriers .....................

Vladimir Scheffer, Partial regularity of solutions to the Navier-Stokes equations.

Eugene Spiegel and Allan Trojan, On semi-simple group algebras. II

Katsuo Takano, On Cameron and Storvick's operator valued function space integral 